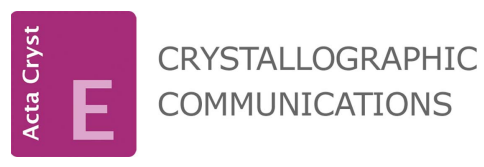

ISSN 2056-9890

Received 2 March 2016

Accepted 31 March 2016

Edited by A. Van der Lee, Université de Montpellier II, France

Keywords: crystal structure; nickel arsenate; ceramic synthesis.

Supporting information: this article has supporting information at journals.iucr.org/e

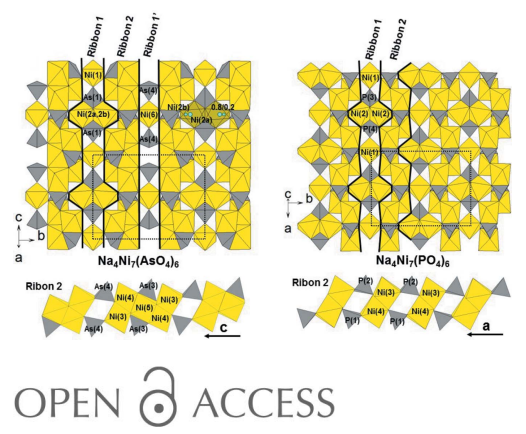

\section{Synthesis and crystal structure of $\mathrm{Na}_{4} \mathrm{Ni}_{7}\left(\mathrm{AsO}_{4}\right)_{6}$}

\author{
Rénald David*
}

Laboratoire de Réactivité et Chimie des Solides, Université de Picardie Jules Verne, CNRS UMR 7314, 33 rue Saint Leu, 80039 Amiens, France. *Correspondence e-mail: renald.david@u-picardie.fr

The title compound, tetrasodium heptanickel hexaarsenate, was obtained by ceramic synthesis and crystallizes in the monoclinic space group $\mathrm{C} 2 / \mathrm{m}$. The asymmetric unit contains seven $\mathrm{Ni}$ atoms of which two have site symmetry $2 / \mathrm{m}$ and three site symmetry 2 , four As atoms of which two have site symmetry $m$ and two site symmetry 2, three $\mathrm{Na}$ atoms of which two have site symmetry 2, and fifteen $\mathrm{O}$ atoms of which four have site symmetry $\mathrm{m}$. The structure of $\mathrm{Na}_{4} \mathrm{Ni}_{7}\left(\mathrm{AsO}_{4}\right)_{6}$ is made of layers of Ni octahedra and As tetrahedra assembled in sheets parallel to the $b c$ plane. These layers are interconnected by cornersharing between $\mathrm{NiO}_{6}$ octahedra and $\mathrm{AsO}_{4}$ tetrahedra. This linkage creates tunnels running along the $c$ axis in which the $\mathrm{Na}$ atoms are located. This arrangement is similar to the one observed in $\mathrm{Na}_{4} \mathrm{Ni}_{7}\left(\mathrm{PO}_{4}\right)_{6}$, but the layers of the two compounds are slightly different because of the disorder of one of the $\mathrm{Ni}$ sites in the structure of the title compound.

\section{Chemical context}

Although the structures of transition metal phosphates have been widely investigated during the last decades, very little work has been done on comparable arsenates due to the toxicity of arsenic. The latter phases can exhibit, however, peculiar properties. $\mathrm{BaCo}_{2}\left(\mathrm{AsO}_{4}\right)_{2}$ is a good example of a quasi-2D system with a magnetically frustrated honeycomb lattice (Regnault et al., 1977). $\mathrm{BaCoAs}_{2} \mathrm{O}_{7}$ appears as the first example of a magnetization step promoted by a structural modulation (David et al., 2013a). $\mathrm{LiCoAsO}_{4}$ shows reversible electrochemical activity at high potential (Satya Kishore \& Varadaraju, 2006). Moreover, a recent study reveals the interest of arsenate groups in playing the role of efficient disconnecting units in the magnetic compound $\mathrm{BaCo}_{2}\left(\mathrm{As}_{3} \mathrm{O}_{6}\right)_{2} \cdot \mathrm{H}_{2} \mathrm{O}$, being the first pure inorganic compound with slow spin dynamics (David et al., 2013b). From the crystal chemistry point of view, substitution of phosphate by arsenate gives the possibility of stabilizing new phases. For example, $\mathrm{NaNiPO}_{4}$ crystallizes with the maricite structure (Senthilkumar et al., 2014), whereas $\mathrm{NaNiAsO}_{4}$ has a honeycomb layer structure (Range \& Meister, 1984). In this study, we describe the structure of $\mathrm{Na}_{4} \mathrm{Ni}_{7}\left(\mathrm{AsO}_{4}\right)_{6}$ and compare it with its phosphate analogue.

\section{Structural commentary}

The structure of the title compound $\mathrm{Na}_{4} \mathrm{Ni}_{7}\left(\mathrm{AsO}_{4}\right)_{6}$ is quite similar to the one of the phosphate homologue $\mathrm{Na}_{4} \mathrm{Ni}_{7}\left(\mathrm{PO}_{4}\right)_{6}$. Both are made of interconnected $\mathrm{Ni}_{7}\left(\mathrm{XO}_{4}\right)_{6}$ layers with tunnels in between where the $\mathrm{Na}$ atoms are located, as shown in Fig. 1a. The arrangement of $\mathrm{NiO}_{6}$ and $\mathrm{XO}_{4}$ in the layer is, however, slightly different, as evidenced in Fig. 2. As described 
a)

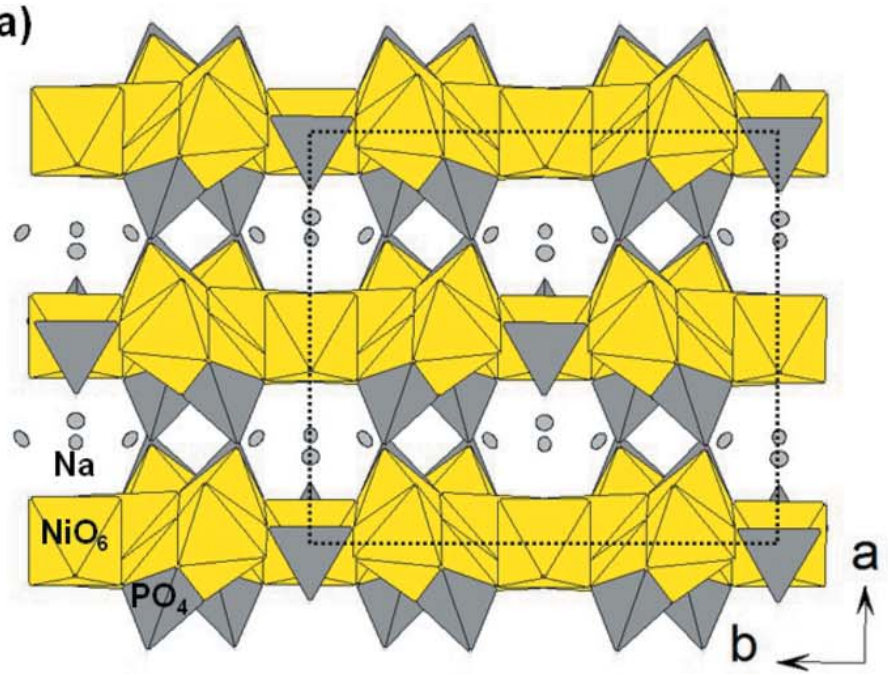

b)

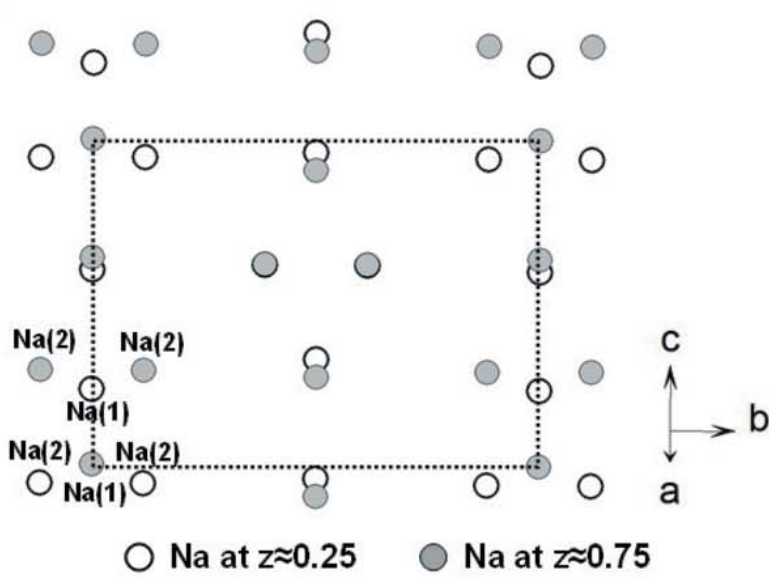

Figure 1

Description of the crystal structure of $\mathrm{Na}_{4} \mathrm{Ni}_{7}\left(\mathrm{AsO}_{4}\right)_{6}$ with $(a)$ a view of the stacking and $(b)$ a view of the Na layers. The dotted lines show the cell edges. Displacement ellipsoids are drawn at the $50 \%$ probability level.

by Moring \& Kostiner (1986), $\mathrm{Na}_{4} \mathrm{Ni}_{7}\left(\mathrm{PO}_{4}\right)_{6}$ layers are made of parallel ribbons (called ribbon 1) containing Ni1, Ni2, P3 and $\mathrm{P} 4$ polyhedra. These ribbons 1 are interconnected by another kind of ribbon (called ribbon 2) made of dimers consisting of edge-sharing $\mathrm{NiO}_{6}$ octahedra (Ni3 and Ni4). The latter are linked to $\mathrm{PO}_{4}$ tetrahedra (P1 and $\left.\mathrm{P} 2\right)$ by edge- and corner-sharing. The difference between the two compounds is associated with the possibility of the $\mathrm{Ni} 2$ atom in
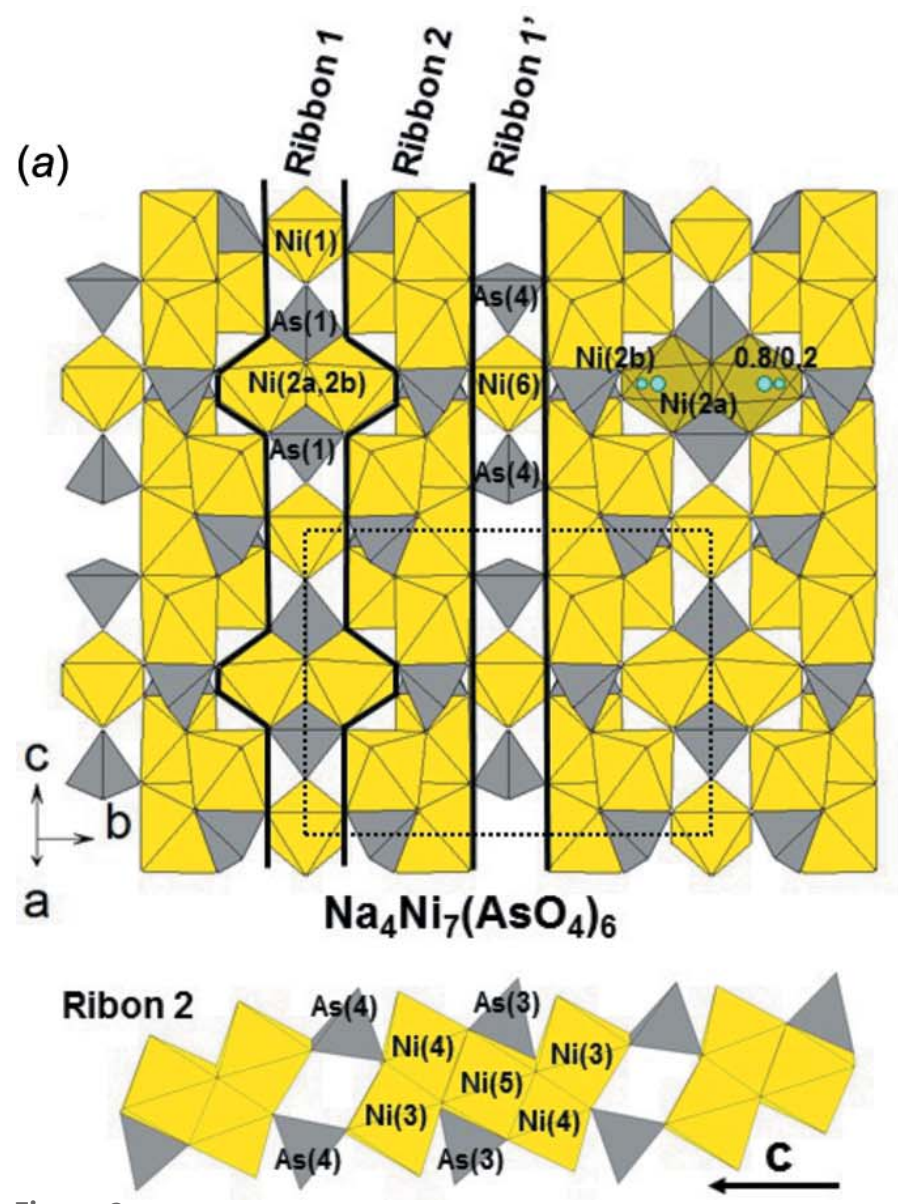

(b)
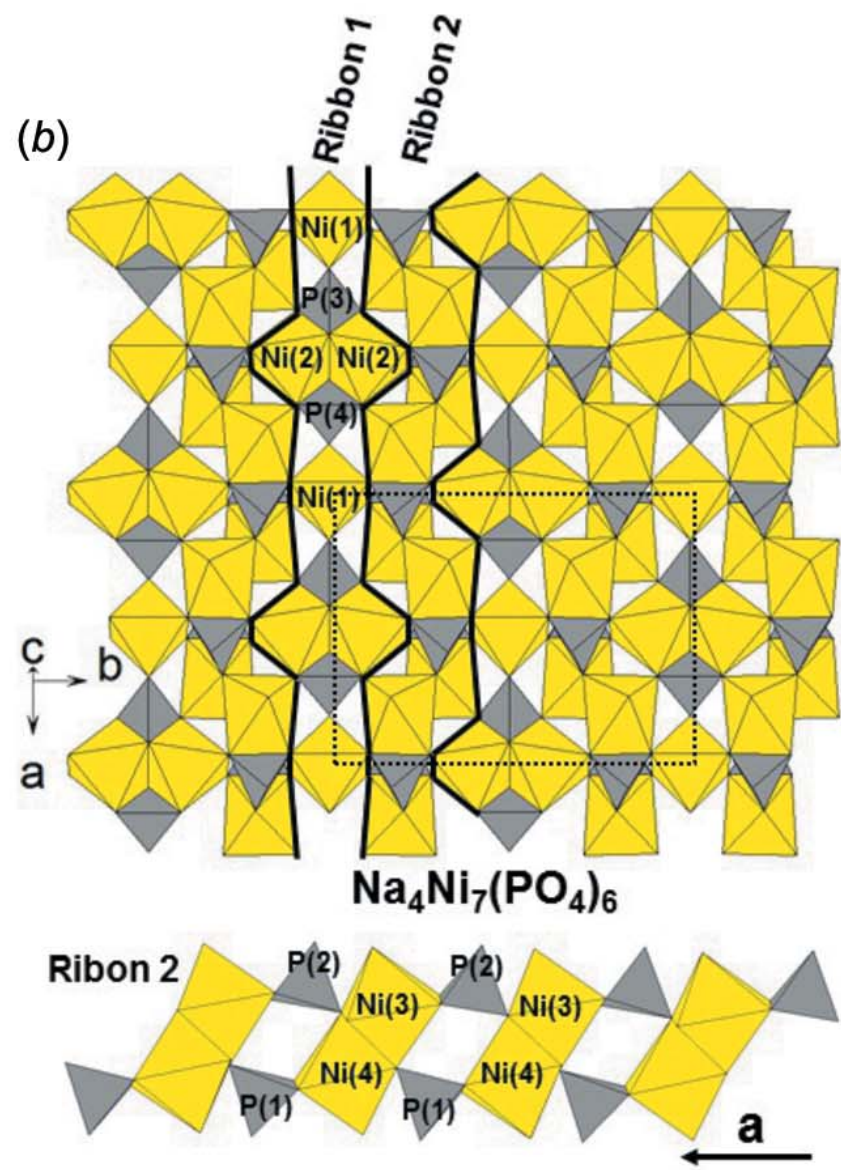

Figure 2

Description of the layers (top) and the ribbon 2 (bottom) of $(a) \mathrm{Na}_{4} \mathrm{Ni}_{7}\left(\mathrm{AsO}_{4}\right)_{6}$ and $(b) \mathrm{Na}_{4} \mathrm{Ni}_{7}\left(\mathrm{PO}_{4}\right)_{6}$. The dotted lines show the cell edges. 
Table 1

Experimental details.

\begin{tabular}{|c|c|}
\hline \multicolumn{2}{|l|}{ Crystal data } \\
\hline Chemical formula & $\mathrm{Na}_{4} \mathrm{Ni}_{7}\left(\mathrm{AsO}_{4}\right)_{6}$ \\
\hline$M_{\mathrm{r}}$ & 1336.3 \\
\hline Crystal system, space group & Monoclinic, $C 2 / m$ \\
\hline Temperature $(\mathrm{K})$ & 293 \\
\hline$a, b, c(\AA)$ & $\begin{array}{l}14.5383(11), 14.5047(11), \\
10.6120(8)\end{array}$ \\
\hline$\beta\left(^{\circ}\right)$ & $118.299(2)$ \\
\hline$V\left(\AA^{3}\right)$ & $1970.3(3)$ \\
\hline$Z$ & 4 \\
\hline Radiation type & Mo $K \alpha$ \\
\hline$\mu\left(\mathrm{mm}^{-1}\right)$ & 16.76 \\
\hline Crystal size (mm) & $0.07 \times 0.06 \times 0.04$ \\
\hline \multicolumn{2}{|l|}{ Data collection } \\
\hline Diffractometer & Bruker D8 Venture \\
\hline Absorption correction & $\begin{array}{l}\text { Multi-scan (SADABS; Bruker, } \\
\text { 2015) }\end{array}$ \\
\hline$T_{\min }, T_{\max }$ & $0.640,0.747$ \\
\hline $\begin{array}{l}\text { No. of measured, independent and } \\
\text { observed }[I>3 \sigma(I)] \text { reflections }\end{array}$ & $48075,3773,2901$ \\
\hline$R_{\mathrm{int}}$ & 0.036 \\
\hline$(\sin \theta / \lambda)_{\max }\left(\AA^{-1}\right)$ & 0.772 \\
\hline \multicolumn{2}{|l|}{ Refinement } \\
\hline$R\left[F^{2}>2 \sigma\left(F^{2}\right)\right], w R\left(F^{2}\right), S$ & $0.039,0.052,2.73$ \\
\hline No. of reflections & 3773 \\
\hline No. of parameters & 146 \\
\hline$\Delta \rho_{\max }, \Delta \rho_{\min }\left(\mathrm{e} \AA^{-3}\right)$ & $3.34,-2.22$ \\
\hline
\end{tabular}

Computer programs: APEX3 and SAINT (Bruker, 2015), SUPERFLIP (Palatinus \& Chapuis, 2007), JANA2006 (Petříčcek et al., 2014, DIAMOND (Brandenburg, 2006) and publCIF (Westrip, 2010).

$\mathrm{Na}_{4} \mathrm{Ni}_{7}\left(\mathrm{PO}_{4}\right)_{6}$ occupying two octahedral sites. The first site, belonging to ribbon 1 , is equivalent to the $\mathrm{Ni} 2 a$ site in $\mathrm{Na}_{4} \mathrm{Ni}_{7}\left(\mathrm{AsO}_{4}\right)_{6}$. The other, equivalent to the $\mathrm{Ni} 2 b$ and $\mathrm{Ni} 5$ sites in $\mathrm{Na}_{4} \mathrm{Ni}_{7}\left(\mathrm{AsO}_{4}\right)_{6}$, belongs to ribbon 2, forming pentamers of edge-sharing $\mathrm{NiO}_{6}$ octahedra. The layers of the title compound $\mathrm{Na}_{4} \mathrm{Ni}_{7}\left(\mathrm{AsO}_{4}\right)_{6}$ can thus be described with three kinds of ribbons, as shown in Fig. 2. The linkage between the layers is done by corner-sharing between $\mathrm{NiO}_{6}$ and $\mathrm{AsO}_{4}$ units of two consecutive ribbons 2 along the stacking axis (Fig. 1a). This linkage is identical to the one of the phosphorus homologue. However, since in $\mathrm{Na}_{4} \mathrm{Ni}_{7}\left(\mathrm{AsO}_{4}\right)_{6}$ layers are made of three different kinds of ribbons, two adjacent layers are shifted to align ribbon 1 with ribbon $1^{\prime}$. That is why in $\mathrm{Na}_{4} \mathrm{Ni}_{7}\left(\mathrm{AsO}_{4}\right)_{6}$ the stacking axis is roughly doubled compared to $\mathrm{Na}_{4} \mathrm{Ni}_{7}\left(\mathrm{PO}_{4}\right)_{6}[c=6.398(2) \AA$ versus $a=14.5383$ (11) $\AA$ in the title structure]. It implies two different kinds of Na layers, as shown in Fig. $1 b$.

\section{Synthesis and crystallization}

Sodium carbonate (>99.5\%), arsenic oxide (99\%) and nickel sulfate hexahydrate $(>99.9 \%)$ were purchased from Sigma-
Aldrich. They were used as received without further purification. Reagents were ground together in stoichiometric ratio in an agate mortar. The obtained mixture was pelletized, placed in an alumina boat and annealed at $573 \mathrm{~K}$ for $1 \mathrm{~h}$. The obtained mixture was reground, pelletized and heated at $1073 \mathrm{~K}\left(5 \mathrm{~K} \mathrm{~min}^{-1}\right)$ for $48 \mathrm{~h}$, after which the alumina boat was removed from the furnace and cooled to room temperature. The brown crystals of the title compound were isolated by hand.

\section{Refinement details}

Crystal data, data collection and structure refinement details are summarized in Table 1 . The (001) reflection, affected by the beamstop, has been removed from the refinement. Another reflection $(\overline{2} 01)$, flagged as potentially affected by the beamstop, was in fact not and was kept in the refinement. After positioning and refining all the atom positions except $\mathrm{Ni} 2 b$, the difference Fourier map revealed residual density ( $\simeq 8 \mathrm{e}^{-3}$ ) near Ni2 $a$ (at $\simeq 0.6 \AA$ ). It was refined introducing a second position $\mathrm{Ni} 2 b$ with complementary occupation. The occupancy ratio was refined to 0.80 (4):0.20 (4) for the Ni2al $\mathrm{Ni} 2 b$ site, constraining the sum to be equal to 1 .

\section{Acknowledgements}

The RS2E (French Network on Electrochemical Energy Storage) and ANR (Labex STORE-EX: grant ANR-10LABX-0076) are acknowledged for funding of the X-ray diffractometer.

\section{References}

Brandenburg, K. (2006). DIAMOND. Crystal Impact GbR, Bonn, Germany.

Bruker (2015). APEX3, SAINT and SADABS. Bruker AXS Inc., Madison, Wisconsin, USA.

David, R., Kabbour, H., Colis, S. \& Mentré, O. (2013a). Inorg. Chem. 52, 13742-13750.

David, R., Kabbour, H., Colis, S., Pautrat, A., Suard, E. \& Mentré, O. (2013b). J. Phys. Chem. C, 117, 18190-18198.

Moring, J. \& Kostiner, E. (1986). J. Solid State Chem. 62, 105-111.

Palatinus, L. \& Chapuis, G. (2007). J. Appl. Cryst. 40, 786-790.

Petrríčcek, V., Dušek, M. \& Palatinus, L. (2014). Z. Kristallogr. 229, 345-352.

Range, K.-J. \& Meister, H. (2014). Z. Naturforsch. Teil B, 39, 118-120.

Regnault, L. P., Burlet, P. \& Rossat-Mignod, J. (1977). Physica B, 8688, 660-662.

Satya Kishore, M. V. V. M. \& Varadaraju, U. V. (2006). Mater. Res. Bull. 41, 601-607.

Senthilkumar, B., Sankar, K. V., Vasylechko, L., Lee, Y.-S. \& Selvan, R. K. (2014). RSC Adv. 4, 53192-53200.

Westrip, S. P. (2010). J. Appl. Cryst. 43, 920-925. 


\section{supporting information}

Acta Cryst. (2016). E72, 632-634 [doi:10.1107/S2056989016005417]

Synthesis and crystal structure of $\mathrm{Na}_{4} \mathrm{Ni}_{7}\left(\mathrm{AsO}_{4}\right)_{6}$

\section{Rénald David}

Computing details

Data collection: APEX3 (Bruker, 2015); cell refinement: SAINT (Bruker, 2015); data reduction: SAINT (Bruker, 2015); program(s) used to solve structure: SUPERFLIP (Palatinus \& Chapuis, 2007); program(s) used to refine structure: JANA2006 (Petrríčcek et al., 2014; molecular graphics: DIAMOND (Brandenburg, 2006); software used to prepare material for publication: publCIF (Westrip, 2010).

Tetrasodium heptanickel hexaarsenate

Crystal data

$\mathrm{Na}_{4} \mathrm{Ni}_{7}\left(\mathrm{AsO}_{4}\right)_{6}$

$M_{r}=1336.3$

Monoclinic, $C 2 / \mathrm{m}$

Hall symbol: $-\mathrm{C} 2 \mathrm{y}$

$a=14.5383$ (11) $\AA$

$b=14.5047$ (11) $\AA$

$c=10.6120(8) \AA$

$\beta=118.299(2)^{\circ}$

$V=1970.3(3) \AA^{3}$

$Z=4$

$F(000)=2520$

$D_{\mathrm{x}}=4.505 \mathrm{Mg} \mathrm{m}^{-3}$

Mo $K \alpha$ radiation, $\lambda=0.71073 \AA$

Cell parameters from 38754 reflections

$\theta=2.1-33.3^{\circ}$

$\mu=16.76 \mathrm{~mm}^{-1}$

$T=293 \mathrm{~K}$

Irregular, brown

$0.07 \times 0.06 \times 0.04 \mathrm{~mm}$

\section{Data collection}

Bruker D8 Venture diffractometer

Radiation source: X-ray tube phi scan

Absorption correction: multi-scan

(SADABS; Bruker, 2015)

$T_{\min }=0.640, T_{\max }=0.747$

48075 measured reflections

3773 independent reflections

2901 reflections with $I>3 \sigma(I)$

$R_{\text {int }}=0.036$

$\theta_{\max }=33.3^{\circ}, \theta_{\min }=2.1^{\circ}$

$h=-21 \rightarrow 20$

$k=-22 \rightarrow 21$

$l=-16 \rightarrow 16$

\section{Refinement}

Refinement on $F$

$R\left[F^{2}>2 \sigma\left(F^{2}\right)\right]=0.039$

$w R\left(F^{2}\right)=0.052$

$S=2.73$

3773 reflections

146 parameters

0 restraints

0 constraints

Weighting scheme based on measured s.u.'s $w=$ $1 /\left(\sigma^{2}(F)+0.0001 F^{2}\right)$

$(\Delta / \sigma)_{\max }=0.015$

$\Delta \rho_{\max }=3.34$ e $\AA^{-3}$

$\Delta \rho_{\min }=-2.22$ e $\AA^{-3}$ 
Fractional atomic coordinates and isotropic or equivalent isotropic displacement parameters $\left(\AA^{2}\right)$

\begin{tabular}{|c|c|c|c|c|c|}
\hline & $x$ & $y$ & $z$ & $U_{\text {iso }} * / U_{\text {eq }}$ & Occ. $(<1)$ \\
\hline Nil & 0.5 & 0 & 0 & 0.0068 (4) & \\
\hline $\mathrm{Ni} 2 \mathrm{a}$ & 0.5 & $0.1306(6)$ & 0.5 & $0.0078(8)$ & $0.80(4)$ \\
\hline $\mathrm{Ni} 2 \mathrm{~b}$ & 0.5 & $0.166(5)$ & 0.5 & $0.027(7)$ & $0.20(4)$ \\
\hline $\mathrm{Ni3}$ & $0.91312(4)$ & $0.18257(3)$ & $0.23065(6)$ & $0.00542(19)$ & \\
\hline $\mathrm{Ni} 4$ & $0.59336(4)$ & $0.18693(3)$ & $0.26615(6)$ & $0.00530(19)$ & \\
\hline $\mathrm{Ni} 5$ & 0.5 & $0.32688(6)$ & 0 & $0.0112(3)$ & \\
\hline Ni6 & 0 & 0 & 0.5 & $0.0061(4)$ & \\
\hline As 1 & $0.85957(3)$ & $0.18473(3)$ & $0.45674(4)$ & $0.00503(16)$ & \\
\hline As2 & $0.65106(3)$ & $0.18354(3)$ & $0.05278(4)$ & $0.00470(15)$ & \\
\hline As3 & $0.47038(5)$ & 0 & $0.28662(6)$ & $0.0062(2)$ & \\
\hline As4 & $0.45990(5)$ & 0.5 & $-0.20991(6)$ & $0.0056(2)$ & \\
\hline $\mathrm{Na} 1$ & $0.2934(2)$ & 0.5 & -0.0189 & $0.0304(13)$ & \\
\hline $\mathrm{Na} 2$ & $0.25173(16)$ & $0.11586(14)$ & $0.3367(2)$ & $0.0305(9)$ & \\
\hline $\mathrm{Na} 3$ & $0.7399(2)$ & 0 & $0.3103(4)$ & $0.0435(15)$ & \\
\hline $\mathrm{O} 1$ & $0.6079(2)$ & 0.09855 (19) & $0.1237(3)$ & $0.0067(5)^{*}$ & \\
\hline $\mathrm{O} 2$ & $0.8783(2)$ & $0.2776(2)$ & $0.3717(3)$ & $0.0125(6)^{*}$ & \\
\hline $\mathrm{O} 3$ & $0.4137(3)$ & 0 & 0.1101 & $0.0104(8)^{*}$ & \\
\hline $\mathrm{O} 4$ & $0.4391(2)$ & 0.21767 (19) & $0.1132(3)$ & $0.0090(6)^{*}$ & \\
\hline O5 & $0.7665(2)$ & $0.1552(2)$ & 0.0682 & $0.0097(6)^{*}$ & \\
\hline O6 & $0.5380(2)$ & $0.4081(2)$ & -0.1240 & $0.0096(6)^{*}$ & \\
\hline $\mathrm{O} 8$ & $0.3535(3)$ & 0.5 & $-0.1899(5)$ & $0.0140(9)^{*}$ & \\
\hline O9 & $0.3901(4)$ & 0 & $0.3581(5)$ & $0.0232(11)^{*}$ & \\
\hline $\mathrm{O} 10$ & $0.9333(2)$ & 0.20454 (19) & 0.6329 & $0.0089(6)^{*}$ & \\
\hline O11 & $0.5468(2)$ & $0.0939(2)$ & 0.3604 & $0.0115(6)^{*}$ & \\
\hline $\mathrm{O} 12$ & $0.7395(2)$ & $0.1588(2)$ & $0.4274(3)$ & $0.0096(6)^{*}$ & \\
\hline $\mathrm{O} 13$ & $0.6448(2)$ & $0.27433(19)$ & $0.1522(3)$ & $0.0092(6)^{*}$ & \\
\hline $\mathrm{O} 14$ & $0.4220(3)$ & 0.5 & $-0.3841(4)$ & $0.0108(9)^{*}$ & \\
\hline $\mathrm{O} 15$ & $0.8965(2)$ & $0.10027(18)$ & 0.3788 & $0.0062(5)^{*}$ & \\
\hline
\end{tabular}

Atomic displacement parameters $\left(\AA^{2}\right)$

\begin{tabular}{lllllll}
\hline & $U^{11}$ & $U^{22}$ & $U^{33}$ & $U^{12}$ & $U^{13}$ & $U^{23}$ \\
\hline Ni1 & $0.0070(5)$ & $0.0067(5)$ & $0.0068(5)$ & 0 & $0.0032(4)$ & 0 \\
Ni2a & $0.0102(7)$ & $0.006(2)$ & $0.0096(7)$ & 0 & $0.0068(5)$ & 0 \\
Ni2b & $0.021(3)$ & $0.04(2)$ & $0.019(3)$ & 0 & $0.010(2)$ & 0 \\
Ni3 & $0.0047(2)$ & $0.0062(3)$ & $0.0048(3)$ & $-0.00029(18)$ & $0.0018(2)$ & $0.00088(18)$ \\
Ni4 & $0.0050(2)$ & $0.0057(3)$ & $0.0048(3)$ & $0.00053(18)$ & $0.0020(2)$ & $-0.00048(18)$ \\
Ni5 & $0.0097(4)$ & $0.0149(4)$ & $0.0084(4)$ & 0 & $0.0039(3)$ & 0 \\
Ni6 & $0.0070(5)$ & $0.0048(5)$ & $0.0066(5)$ & 0 & $0.0033(4)$ & 0 \\
As1 & $0.0049(2)$ & $0.0058(2)$ & $0.0047(2)$ & $0.00044(14)$ & $0.00248(16)$ & $0.00007(14)$ \\
As2 & $0.00360(19)$ & $0.0057(2)$ & $0.0046(2)$ & $0.00079(14)$ & $0.00184(16)$ & $0.00099(14)$ \\
As3 & $0.0082(3)$ & $0.0051(3)$ & $0.0054(3)$ & 0 & $0.0032(2)$ & 0 \\
As4 & $0.0061(3)$ & $0.0044(3)$ & $0.0058(3)$ & 0 & $0.0024(2)$ & 0 \\
Na1 & $0.0270(17)$ & $0.0353(17)$ & $0.0297(17)$ & 0 & $0.0141(14)$ & 0 \\
Na2 & $0.0251(11)$ & $0.0240(11)$ & $0.0399(14)$ & $-0.0090(9)$ & $0.0134(10)$ & $-0.0080(10)$
\end{tabular}


0

Geometric parameters $\left(\AA,{ }^{\circ}\right)$

\begin{tabular}{|c|c|c|c|}
\hline $\mathrm{Ni1}-\mathrm{O} 1$ & $2.068(2)$ & $\mathrm{Ni6}-\mathrm{O} 15^{\mathrm{iv}}$ & $2.049(2)$ \\
\hline $\mathrm{Ni} 1-\mathrm{O} 1^{\mathrm{i}}$ & $2.068(2)$ & $\mathrm{Ni6}-\mathrm{O} 15^{\text {xiii }}$ & 2.049 (2) \\
\hline $\mathrm{Ni} 1-\mathrm{O} 1^{\mathrm{ii}}$ & $2.068(2)$ & $\mathrm{Ni6}-\mathrm{O} 15^{\mathrm{xiv}}$ & $2.049(2)$ \\
\hline $\mathrm{Ni1}-\mathrm{O} 1^{\mathrm{iii}}$ & $2.068(2)$ & As $1-\mathrm{Na} 2^{\mathrm{vii}}$ & $3.249(2)$ \\
\hline $\mathrm{Ni} 1-\mathrm{O} 3$ & $2.081(6)$ & As $1-\mathrm{Na} 3$ & $3.1713(16)$ \\
\hline $\mathrm{Ni} 1-\mathrm{O}^{\mathrm{i}}$ & $2.081(6)$ & As $1-\mathrm{O} 2$ & $1.714(3)$ \\
\hline $\mathrm{Ni} 2 \mathrm{a}-\mathrm{Ni} 2 \mathrm{~b}$ & $0.51(7)$ & As $1-\mathrm{O} 10$ & $1.681(3)$ \\
\hline $\mathrm{Ni} 2 \mathrm{a}-\mathrm{Na} 2$ & $3.185(2)$ & As1-O12 & $1.664(3)$ \\
\hline $\mathrm{Ni} 2 \mathrm{a}-\mathrm{Na} 2^{\mathrm{iv}}$ & $3.185(2)$ & As $1-\mathrm{O} 15$ & $1.702(3)$ \\
\hline $\mathrm{Ni} 2 \mathrm{a}-\mathrm{O} 11$ & $1.974(4)$ & As $2-\mathrm{O} 1$ & $1.711(3)$ \\
\hline $\mathrm{Ni} 2 \mathrm{a}-\mathrm{O} 11^{\mathrm{iv}}$ & $1.974(4)$ & $\mathrm{As} 2-\mathrm{O} 4^{\mathrm{i}}$ & $1.696(3)$ \\
\hline $\mathrm{Ni} 2 \mathrm{~b}-\mathrm{Na} 2$ & $3.260(17)$ & As $2-\mathrm{O} 5$ & $1.659(3)$ \\
\hline $\mathrm{Ni} 2 \mathrm{~b}-\mathrm{Na} 2^{\mathrm{iv}}$ & $3.260(17)$ & As2-O13 & $1.716(3)$ \\
\hline $\mathrm{Ni} 2 \mathrm{~b}-\mathrm{O} 2^{\mathrm{v}}$ & $1.83(3)$ & As3-O3 & $1.650(4)$ \\
\hline $\mathrm{Ni} 2 \mathrm{~b}-\mathrm{O} 2^{\mathrm{vi}}$ & $1.83(3)$ & As $3-O 9$ & $1.667(7)$ \\
\hline $\mathrm{Ni} 3-\mathrm{O} 4^{\mathrm{vii}}$ & $2.058(3)$ & As3-O11 & $1.697(3)$ \\
\hline $\mathrm{Ni} 3-\mathrm{O} 5$ & 2.047 (3) & As3-O11 $1^{\mathrm{iii}}$ & $1.697(3)$ \\
\hline $\mathrm{Ni3}-\mathrm{O}^{\text {viii }}$ & $2.069(3)$ & $\mathrm{As} 4-\mathrm{Na} 1^{\mathrm{i}}$ & $3.240(3)$ \\
\hline $\mathrm{Ni3}-\mathrm{O} 10^{\text {ix }}$ & $2.029(3)$ & As4- $\mathrm{Na}^{\mathrm{xv}}$ & $3.190(2)$ \\
\hline $\mathrm{Ni3}-\mathrm{O} 15$ & $2.077(3)$ & As4-Na2 ${ }^{\text {xvi }}$ & $3.190(2)$ \\
\hline $\mathrm{Ni4}-\mathrm{O} 1$ & $2.068(3)$ & As4-O6 & 1.707 (3) \\
\hline $\mathrm{Ni} 4-\mathrm{O} 4$ & $2.101(3)$ & As4-O $6^{\mathrm{xvii}}$ & 1.707 (3) \\
\hline $\mathrm{Ni} 4-\mathrm{O} 10^{v}$ & $2.042(3)$ & As $4-08$ & $1.658(6)$ \\
\hline $\mathrm{Ni4}-\mathrm{O} 11$ & $1.980(4)$ & As4-O14 & $1.660(5)$ \\
\hline $\mathrm{Ni4}-\mathrm{O} 12$ & $2.041(3)$ & $\mathrm{Na} 1-\mathrm{Na} 2^{\mathrm{xv}}$ & $3.540(4)$ \\
\hline $\mathrm{Ni} 5-\mathrm{O} 6$ & $2.029(3)$ & $\mathrm{Na} 1-\mathrm{Na} 2^{\text {xvi }}$ & $3.540(4)$ \\
\hline $\mathrm{Ni} 5-\mathrm{O}^{\mathrm{i}}$ & $2.029(3)$ & $\mathrm{Na} 1-\mathrm{Na} 3^{\mathrm{xviii}}$ & $3.923(6)$ \\
\hline $\mathrm{Ni5}-\mathrm{O} 13$ & $2.097(3)$ & $\mathrm{Na} 1-\mathrm{O} 8$ & $2.358(7)$ \\
\hline $\mathrm{Ni} 5-\mathrm{O} 13^{\mathrm{i}}$ & $2.097(3)$ & $\mathrm{Na} 2-\mathrm{Na} 2^{\mathrm{iii}}$ & $3.361(3)$ \\
\hline $\mathrm{Ni6}-\mathrm{O} 14^{\mathrm{x}}$ & $2.030(6)$ & $\mathrm{Na} 2-\mathrm{O} 2^{\mathrm{vi}}$ & $2.294(4)$ \\
\hline $\mathrm{Ni} 6-\mathrm{O} 14^{\mathrm{xi}}$ & $2.030(6)$ & $\mathrm{Na} 2-\mathrm{O}^{\mathrm{xi}}$ & $2.309(3)$ \\
\hline $\mathrm{Ni6}-\mathrm{O} 15^{\mathrm{xii}}$ & $2.049(2)$ & $\mathrm{Na} 2-\mathrm{O} 13^{\mathrm{vi}}$ & $2.429(3)$ \\
\hline $\mathrm{O} 1-\mathrm{Ni} 1-\mathrm{O} 1^{\mathrm{i}}$ & $92.53(10)$ & $\mathrm{Na} 1^{\mathrm{i}}-\mathrm{As} 4-\mathrm{Na} 2^{\mathrm{xvi}}$ & $145.04(5)$ \\
\hline $\mathrm{O} 1-\mathrm{Ni} 1-\mathrm{O} 1^{\mathrm{ii}}$ & $180.0(5)$ & 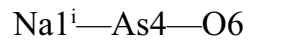 & $51.71(9)$ \\
\hline $\mathrm{O} 1-\mathrm{Ni} 1-\mathrm{O} 1^{\mathrm{iii}}$ & $87.47(10)$ & 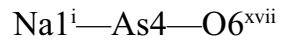 & $51.71(9)$ \\
\hline $\mathrm{O} 1-\mathrm{Ni1}-\mathrm{O} 3$ & $96.97(12)$ & 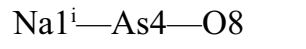 & $132.24(16)$ \\
\hline $\mathrm{O} 1-\mathrm{Ni} 1-\mathrm{O} 3^{\mathrm{i}}$ & $83.03(12)$ & 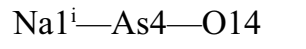 & $119.95(18)$ \\
\hline 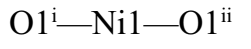 & $87.47(10)$ & $\mathrm{Na} 2^{\mathrm{xv}}-\mathrm{As} 4-\mathrm{Na} 2^{\mathrm{xvi}}$ & $63.58(5)$ \\
\hline 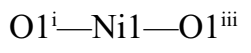 & $180.0(5)$ & $\mathrm{Na} 2^{\mathrm{xv}}-\mathrm{As} 4-\mathrm{O} 6$ & $154.25(13)$ \\
\hline $\mathrm{O} 11^{\mathrm{i}-\mathrm{Ni} 1-\mathrm{O} 3}$ & $83.03(12)$ & $\mathrm{Na} 2^{\mathrm{xv}}-\mathrm{As} 4-\mathrm{O}^{\mathrm{xvii}}$ & $94.48(10)$ \\
\hline $\mathrm{O} 1^{\mathrm{i}}-\mathrm{Ni} 1-\mathrm{O} 3^{\mathrm{i}}$ & $96.97(12)$ & $\mathrm{Na} 2^{\mathrm{xv}}-\mathrm{As} 4-\mathrm{O} 8$ & $44.13(10)$ \\
\hline $\mathrm{O} 1 \mathrm{ii}-\mathrm{Ni} 1-\mathrm{O} 1^{\mathrm{iii}}$ & $92.53(10)$ & $\mathrm{Na} 2^{\mathrm{xv}}-\mathrm{As} 4-\mathrm{O} 14$ & $77.65(15)$ \\
\hline $\mathrm{O} 1{ }^{\mathrm{ii}}-\mathrm{Ni1}-\mathrm{O} 3$ & $83.03(12)$ & $\mathrm{Na} 2^{\mathrm{xvi}}-\mathrm{As} 4-\mathrm{O} 6$ & $94.48(10)$ \\
\hline
\end{tabular}




\begin{tabular}{|c|c|}
\hline $\mathrm{O} 1^{\mathrm{ii}}-\mathrm{Ni} 1-\mathrm{O} 3^{\mathrm{i}}$ & $96.97(12)$ \\
\hline $\mathrm{O} 11^{\mathrm{ii}}-\mathrm{Ni} 1-\mathrm{O} 3$ & $96.97(12)$ \\
\hline 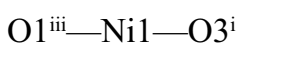 & $83.03(12)$ \\
\hline $\mathrm{O} 3-\mathrm{Ni} 1-\mathrm{O} 3^{\mathrm{i}}$ & $180.0(5)$ \\
\hline $\mathrm{Ni} 2 \mathrm{~b}-\mathrm{Ni} 2 \mathrm{a}-\mathrm{Na} 2$ & $93.85(16)$ \\
\hline $\mathrm{Ni} 2 \mathrm{~b}-\mathrm{Ni} 2 \mathrm{a}-\mathrm{Na} 2^{\mathrm{iv}}$ & $93.85(16)$ \\
\hline $\mathrm{Ni} 2 \mathrm{~b}-\mathrm{Ni} 2 \mathrm{a}-\mathrm{O} 11$ & $105.6(3)$ \\
\hline $\mathrm{Ni} 2 \mathrm{~b}-\mathrm{Ni} 2 \mathrm{a}-\mathrm{O} 11^{\mathrm{iv}}$ & $105.6(3)$ \\
\hline $\mathrm{Na} 2-\mathrm{Ni} 2 \mathrm{a}-\mathrm{Na} 2^{\mathrm{iv}}$ & $172.3(3)$ \\
\hline $\mathrm{Na} 2-\mathrm{Ni} 2 \mathrm{a}-\mathrm{O} 11$ & $106.17(12)$ \\
\hline $\mathrm{Na} 2-\mathrm{Ni} 2 \mathrm{a}-\mathrm{O} 11^{\mathrm{iv}}$ & $71.66(9)$ \\
\hline $\mathrm{Na} 2^{\mathrm{iv}}-\mathrm{Ni} 2 \mathrm{a}-\mathrm{O} 11$ & $71.66(9)$ \\
\hline $\mathrm{Na} 2^{\mathrm{iv}}-\mathrm{Ni} 2 \mathrm{a}-\mathrm{O} 11^{\mathrm{iv}}$ & $106.17(12)$ \\
\hline $\mathrm{O} 11-\mathrm{Ni} 2 \mathrm{a}-\mathrm{O} 11^{\mathrm{iv}}$ & $148.7(5)$ \\
\hline $\mathrm{Ni} 2 \mathrm{a}-\mathrm{Ni} 2 \mathrm{~b}-\mathrm{Na} 2$ & $77.1(13)$ \\
\hline $\mathrm{Ni} 2 \mathrm{a}-\mathrm{Ni} 2 \mathrm{~b}-\mathrm{Na} 2^{\mathrm{iv}}$ & $77.1(13)$ \\
\hline $\mathrm{Ni} 2 \mathrm{a}-\mathrm{Ni} 2 \mathrm{~b}-\mathrm{O} 2^{\mathrm{v}}$ & $116(2)$ \\
\hline $\mathrm{Ni} 2 \mathrm{a}-\mathrm{Ni} 2 \mathrm{~b}-\mathrm{O} 2^{\mathrm{vi}}$ & $116(2)$ \\
\hline $\mathrm{Na} 2-\mathrm{Ni} 2 \mathrm{~b}-\mathrm{Na} 2^{\mathrm{iv}}$ & $154(3)$ \\
\hline $\mathrm{Na} 2-\mathrm{Ni} 2 \mathrm{~b}-\mathrm{O} 2^{\mathrm{v}}$ & $158(2)$ \\
\hline $\mathrm{Na} 2-\mathrm{Ni} 2 \mathrm{~b}-\mathrm{O} 2^{\mathrm{vi}}$ & $43.1(7)$ \\
\hline $\mathrm{Na} 2^{\mathrm{iv}}-\mathrm{Ni} 2 \mathrm{~b}-\mathrm{O} 2^{\mathrm{v}}$ & $43.1(7)$ \\
\hline $\mathrm{Na} 2^{\mathrm{iv}}-\mathrm{Ni} 2 \mathrm{~b}-\mathrm{O} 2^{\mathrm{vi}}$ & $158(2)$ \\
\hline $\mathrm{O} 2^{\mathrm{v}}-\mathrm{Ni} 2 \mathrm{~b}-\mathrm{O} 2^{\mathrm{vi}}$ & $127(4)$ \\
\hline $\mathrm{O} 4{ }^{\mathrm{vii}}-\mathrm{Ni} 3-\mathrm{O} 5$ & $92.46(12)$ \\
\hline $\mathrm{O} 4^{\mathrm{vii}-\mathrm{Ni} 3-\mathrm{O}^{\mathrm{viii}}}$ & $84.64(13)$ \\
\hline $\mathrm{O} 4^{\mathrm{vii}}-\mathrm{Ni} 3-\mathrm{O} 10^{\mathrm{ix}}$ & $82.32(12)$ \\
\hline $\mathrm{O} 4^{\mathrm{vii}}-\mathrm{Ni} 3-\mathrm{O} 15$ & $169.39(11)$ \\
\hline $\mathrm{O} 5-\mathrm{Ni} 3-\mathrm{O} 6^{\mathrm{viii}}$ & $84.66(12)$ \\
\hline $\mathrm{O} 5-\mathrm{Ni3}-\mathrm{O} 10^{\mathrm{ix}}$ & $170.33(16)$ \\
\hline $\mathrm{O} 5-\mathrm{Ni3}-\mathrm{O} 15$ & $94.46(12)$ \\
\hline 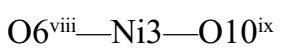 & $86.74(12)$ \\
\hline $\mathrm{O}^{\mathrm{vii}}-\mathrm{Ni} 3-\mathrm{O} 15$ & $103.99(12)$ \\
\hline $\mathrm{O} 10^{\mathrm{ix}}-\mathrm{Ni3}-\mathrm{O} 15$ & $91.91(12)$ \\
\hline $\mathrm{O} 1-\mathrm{Ni} 4-\mathrm{O} 4$ & $90.49(11)$ \\
\hline $\mathrm{O} 1-\mathrm{Ni} 4-\mathrm{O} 10^{\mathrm{v}}$ & $166.43(12)$ \\
\hline $\mathrm{O} 1-\mathrm{Ni4}-\mathrm{O} 11$ & $97.17(13)$ \\
\hline $\mathrm{O} 1-\mathrm{Ni} 4-\mathrm{O} 12$ & $93.57(12)$ \\
\hline $\mathrm{O} 4-\mathrm{Ni} 4-\mathrm{O} 10^{\mathrm{v}}$ & $80.96(11)$ \\
\hline $\mathrm{O} 4-\mathrm{Ni} 4-\mathrm{O} 11$ & $92.27(12)$ \\
\hline $\mathrm{O} 4-\mathrm{Ni} 4-\mathrm{O} 12$ & $175.26(15)$ \\
\hline $\mathrm{O} 10$ - $-\mathrm{Ni} 4-\mathrm{O} 11$ & $93.70(14)$ \\
\hline $\mathrm{O} 10^{\mathrm{v}}-\mathrm{Ni} 4-\mathrm{O} 12$ & $95.48(12)$ \\
\hline $\mathrm{O} 11-\mathrm{Ni4}-\mathrm{O} 12$ & $84.81(12)$ \\
\hline $\mathrm{O} 6-\mathrm{Ni} 5-\mathrm{O}^{\mathrm{i}}$ & $108.95(13)$ \\
\hline $\mathrm{O} 6-\mathrm{Ni} 5-\mathrm{O} 13$ & $103.20(12)$ \\
\hline $\mathrm{O} 6-\mathrm{Ni5}-\mathrm{O} 13^{\mathrm{i}}$ & $101.19(12)$ \\
\hline $\mathrm{O} 6-\mathrm{i}-\mathrm{Ni} 5-\mathrm{O} 13$ & $101.19(12)$ \\
\hline
\end{tabular}

\begin{tabular}{|c|c|}
\hline $\mathrm{Na} 2^{\mathrm{xvi}}-\mathrm{As} 4-\mathrm{O}^{\mathrm{xvii}}$ & $154.25(13)$ \\
\hline $\mathrm{Na} 2^{\mathrm{xvi}}-\mathrm{As} 4-\mathrm{O} 8$ & $44.13(10)$ \\
\hline $\mathrm{Na} 2^{\mathrm{xvi}}-\mathrm{As} 4-\mathrm{O} 14$ & $77.65(15)$ \\
\hline $\mathrm{O} 6-\mathrm{As} 4-\mathrm{O}^{\mathrm{xvii}}$ & $102.64(12)$ \\
\hline $\mathrm{O} 6-\mathrm{As} 4-\mathrm{O} 8$ & $110.86(15)$ \\
\hline $\mathrm{O} 6-\mathrm{As} 4-\mathrm{O} 14$ & $112.34(14)$ \\
\hline $\mathrm{O}^{\mathrm{xvi}}-\mathrm{As} 4-\mathrm{O} 8$ & $110.86(15)$ \\
\hline $\mathrm{O}^{\mathrm{xvii}}-\mathrm{As} 4-\mathrm{O} 14$ & $112.34(14)$ \\
\hline $\mathrm{O} 8-\mathrm{As} 4-\mathrm{O} 14$ & $107.8(2)$ \\
\hline $\mathrm{As} 4^{\mathrm{i}}-\mathrm{Na} 1-\mathrm{Na} 2^{\mathrm{xv}}$ & 110.70 \\
\hline $\mathrm{As} 4^{\mathrm{i}}-\mathrm{Na} 1-\mathrm{Na} 2^{\mathrm{xvi}}$ & $110.70(11)$ \\
\hline $\mathrm{As} 4^{\mathrm{i}}-\mathrm{Na} 1-\mathrm{Na} 3^{\text {xviii }}$ & $87.08(8)$ \\
\hline $\mathrm{As} 4 \mathrm{i}-\mathrm{Na} 1-\mathrm{O} 8$ & $83.94(14)$ \\
\hline $\mathrm{Na} 2^{\mathrm{xv}}-\mathrm{Na} 1-\mathrm{Na} 2^{\mathrm{xvi}}$ & $56.69(7)$ \\
\hline $\mathrm{Na} 2^{\mathrm{xv}}-\mathrm{Na} 1-\mathrm{Na} 3^{\text {xviii }}$ & $145.38(8)$ \\
\hline $\mathrm{Na} 2^{\mathrm{xv}}-\mathrm{Na} 1-\mathrm{O} 8$ & $40.16(9)$ \\
\hline $\mathrm{Na} 2^{\text {xvi }}-\mathrm{Na} 1-\mathrm{Na} 3^{\text {xviii }}$ & $145.38(8)$ \\
\hline 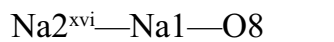 & $40.16(9)$ \\
\hline $\mathrm{Na} 3^{\text {xviii }-\mathrm{Na} 1-\mathrm{O} 8}$ & $171.02(14)$ \\
\hline $\mathrm{Ni} 2 \mathrm{a}-\mathrm{Na} 2-\mathrm{Ni} 2 \mathrm{~b}$ & $9.1(13)$ \\
\hline $\mathrm{Ni} 2 \mathrm{a}-\mathrm{Na} 2-\mathrm{As} 1^{\mathrm{vi}}$ & $61.00(16)$ \\
\hline $\mathrm{Ni} 2 \mathrm{a}-\mathrm{Na} 2-\mathrm{As} 4^{\mathrm{xi}}$ & $151.93(17)$ \\
\hline $\mathrm{Ni} 2 \mathrm{a}-\mathrm{Na} 2-\mathrm{Na} 1^{\mathrm{xi}}$ & $101.59(12)$ \\
\hline $\mathrm{Ni} 2 \mathrm{a}-\mathrm{Na} 2-\mathrm{Na} 2^{\mathrm{iii}}$ & $93.85(17)$ \\
\hline $\mathrm{Ni} 2 \mathrm{a}-\mathrm{Na} 2-\mathrm{O} 2^{\mathrm{vi}}$ & $41.55(17)$ \\
\hline $\mathrm{Ni} 2 \mathrm{a}-\mathrm{Na} 2-\mathrm{O} 8^{\mathrm{xi}}$ & $129.3(2)$ \\
\hline $\mathrm{Ni} 2 \mathrm{a}-\mathrm{Na} 2-\mathrm{O} 13^{\mathrm{vi}}$ & $121.44(17)$ \\
\hline $\mathrm{Ni} 2 \mathrm{~b}-\mathrm{Na} 2-\mathrm{As} 1^{\mathrm{vi}}$ & $52.1(12)$ \\
\hline $\mathrm{Ni} 2 \mathrm{~b}-\mathrm{Na} 2-\mathrm{As} 4^{\mathrm{xi}}$ & $160.9(13)$ \\
\hline $\mathrm{Ni} 2 \mathrm{~b}-\mathrm{Na} 2-\mathrm{Na} 1^{\mathrm{xi}}$ & $105.7(6)$ \\
\hline $\mathrm{Ni} 2 \mathrm{~b}-\mathrm{Na} 2-\mathrm{Na} 2^{\mathrm{iii}}$ & $102.9(13)$ \\
\hline $\mathrm{Ni} 2 \mathrm{~b}-\mathrm{Na} 2-\mathrm{O} 2^{\mathrm{vi}}$ & $33.1(12)$ \\
\hline $\mathrm{Ni} 2 \mathrm{~b}-\mathrm{Na} 2-\mathrm{O} 8^{\mathrm{xi}}$ & $137.2(11)$ \\
\hline $\mathrm{Ni} 2 \mathrm{~b}-\mathrm{Na} 2-\mathrm{O} 13^{\mathrm{vi}}$ & $114.0(11)$ \\
\hline 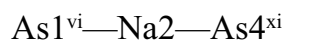 & $145.50(8)$ \\
\hline 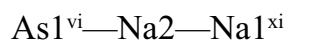 & $129.31(10)$ \\
\hline 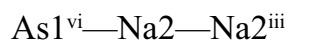 & $152.92(7)$ \\
\hline 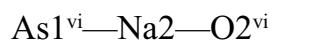 & $30.22(10)$ \\
\hline 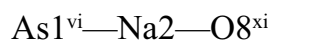 & $162.71(11)$ \\
\hline 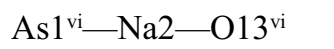 & $74.79(8)$ \\
\hline $\mathrm{As} 4^{\mathrm{xi}}-\mathrm{Na} 2-\mathrm{Na} 1^{\mathrm{xi}}$ & $69.02(7)$ \\
\hline $\mathrm{As} 4^{\mathrm{xi}}-\mathrm{Na} 2-\mathrm{Na} 2^{\mathrm{iii}}$ & $58.21(5)$ \\
\hline $\mathrm{As} 4^{\mathrm{xi}}-\mathrm{Na} 2-\mathrm{O} 2^{\mathrm{vi}}$ & $164.23(11)$ \\
\hline 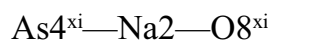 & $30.00(15)$ \\
\hline 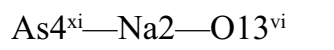 & $83.39(9)$ \\
\hline $\mathrm{Na} 1^{\mathrm{xi}}-\mathrm{Na} 2-\mathrm{Na} 2^{\mathrm{iii}}$ & $61.65(6)$ \\
\hline $\mathrm{Na} 1^{\mathrm{xi}}-\mathrm{Na} 2-\mathrm{O} 2^{\mathrm{vi}}$ & $104.27(14)$ \\
\hline $\mathrm{Na} 1^{\mathrm{xi}}-\mathrm{Na} 2-\mathrm{O} 8^{\mathrm{xi}}$ & $41.18(16)$ \\
\hline
\end{tabular}




\begin{tabular}{|c|c|}
\hline $\mathrm{O}^{\mathrm{i}}-\mathrm{Ni} 5-\mathrm{O}^{\mathrm{i}}$ & $103.20(12)$ \\
\hline $\mathrm{O} 13-\mathrm{Ni} 5-\mathrm{O} 13^{\mathrm{i}}$ & $137.37(11)$ \\
\hline $\mathrm{O} 14^{\mathrm{x}}-\mathrm{Ni6}-\mathrm{O} 14^{\mathrm{xi}}$ & $180.0(5)$ \\
\hline $\mathrm{O} 14^{\mathrm{x}}-\mathrm{Ni} 6-\mathrm{O} 15^{\mathrm{xii}}$ & $85.74(12)$ \\
\hline $\mathrm{O} 14^{\mathrm{x}}-\mathrm{Ni} 6-\mathrm{O} 15^{\mathrm{iv}}$ & $94.26(12)$ \\
\hline $\mathrm{O} 14^{\mathrm{x}}-\mathrm{Ni} 6-\mathrm{O} 15^{\mathrm{xiii}}$ & $94.26(12)$ \\
\hline $\mathrm{O} 14^{\mathrm{x}}-\mathrm{Ni} 6-\mathrm{O} 15^{\mathrm{xiv}}$ & $85.74(12)$ \\
\hline $\mathrm{O} 14^{\mathrm{xi}}-\mathrm{Ni} 6-\mathrm{O} 15^{\mathrm{xii}}$ & $94.26(12)$ \\
\hline $\mathrm{O} 14^{\mathrm{xi}-\mathrm{Ni} 6}-\mathrm{O} 15^{\mathrm{iv}}$ & $85.74(12)$ \\
\hline $\mathrm{O} 14^{\mathrm{xi}}-\mathrm{Ni} 6-\mathrm{O} 15^{\mathrm{xiii}}$ & $85.74(12)$ \\
\hline $\mathrm{O} 14^{\mathrm{xi}}-\mathrm{Ni} 6-\mathrm{O} 15^{\mathrm{xiv}}$ & $94.26(12)$ \\
\hline $\mathrm{O} 15^{\mathrm{xii}}-\mathrm{Ni6}-\mathrm{O} 15^{\mathrm{iv}}$ & $89.57(10)$ \\
\hline $\mathrm{O} 15^{\mathrm{xii}}-\mathrm{Ni} 6-\mathrm{O} 15^{\mathrm{xiii}}$ & $180.0(5)$ \\
\hline $\mathrm{O} 15^{\mathrm{xii}}-\mathrm{Ni} 6-\mathrm{O} 15^{\mathrm{xiv}}$ & $90.43(10)$ \\
\hline $\mathrm{O} 15^{\mathrm{iv}}-\mathrm{Ni6}-\mathrm{O} 15^{\mathrm{xiii}}$ & $90.43(10)$ \\
\hline $\mathrm{O} 15^{\mathrm{iv}}-\mathrm{Ni6}-\mathrm{O} 15^{\mathrm{xiv}}$ & $180.0(5)$ \\
\hline $\mathrm{O} 15^{\mathrm{xiii}}-\mathrm{Ni6}-\mathrm{O} 15^{\mathrm{xiv}}$ & $89.57(10)$ \\
\hline $\mathrm{Na} 2^{\mathrm{vii}}-\mathrm{As} 1-\mathrm{Na} 3$ & $120.62(5)$ \\
\hline $\mathrm{Na} 2^{2 \mathrm{vi}}-\mathrm{As} 1-\mathrm{O} 2$ & $42.36(13)$ \\
\hline $\mathrm{Na} 2^{\mathrm{vii}}-\mathrm{As} 1-\mathrm{O} 10$ & $102.64(10)$ \\
\hline $\mathrm{Na} 2^{\mathrm{vii}}-\mathrm{As} 1-\mathrm{O} 12$ & $81.93(11)$ \\
\hline $\mathrm{Na} 2^{\mathrm{vii}}-\mathrm{As} 1-\mathrm{O} 15$ & $130.93(11)$ \\
\hline $\mathrm{Na} 3-\mathrm{As} 1-\mathrm{O} 2$ & $126.42(12)$ \\
\hline $\mathrm{Na3}-\mathrm{As} 1-\mathrm{O} 10$ & $127.00(12)$ \\
\hline $\mathrm{Na} 3-\mathrm{As} 1-\mathrm{O} 12$ & $55.54(13)$ \\
\hline $\mathrm{Na3}-\mathrm{As} 1-\mathrm{O} 15$ & $51.68(13)$ \\
\hline $\mathrm{O} 2-\mathrm{As} 1-\mathrm{O} 10$ & $105.90(14)$ \\
\hline $\mathrm{O} 2-\mathrm{As} 1-\mathrm{O} 12$ & $119.59(14)$ \\
\hline $\mathrm{O} 2-\mathrm{As} 1-\mathrm{O} 15$ & $98.30(17)$ \\
\hline $\mathrm{O} 10-\mathrm{As} 1-\mathrm{O} 12$ & $107.84(17)$ \\
\hline $\mathrm{O} 10-\mathrm{As} 1-\mathrm{O} 15$ & $118.79(13)$ \\
\hline O12-As1-O15 & $106.93(14)$ \\
\hline $\mathrm{O} 1-\mathrm{As} 2-\mathrm{O} 4^{\mathrm{i}}$ & $113.78(13)$ \\
\hline $\mathrm{O} 1-\mathrm{As} 2-\mathrm{O} 5$ & $110.07(15)$ \\
\hline $\mathrm{O} 1-\mathrm{As} 2-\mathrm{O} 13$ & $98.34(16)$ \\
\hline $\mathrm{O} 4 \mathrm{i}-\mathrm{As} 2-\mathrm{O} 5$ & $114.98(17)$ \\
\hline $\mathrm{O} 4-\mathrm{As} 2-\mathrm{O} 13$ & $100.05(14)$ \\
\hline $\mathrm{O} 5-\mathrm{As} 2-\mathrm{O} 13$ & $118.36(14)$ \\
\hline $\mathrm{O} 3-\mathrm{As} 3-\mathrm{O} 9$ & $115.8(2)$ \\
\hline $\mathrm{O} 3-\mathrm{As} 3-\mathrm{O} 11$ & $112.91(14)$ \\
\hline $\mathrm{O} 3-\mathrm{As} 3-\mathrm{O} 11^{\mathrm{iii}}$ & $112.91(14)$ \\
\hline $\mathrm{O} 9-\mathrm{As} 3-\mathrm{O} 11$ & $103.68(16)$ \\
\hline $\mathrm{O} 9-\mathrm{As} 3-\mathrm{O} 11^{\mathrm{iii}}$ & $103.68(16)$ \\
\hline $\mathrm{O} 11-\mathrm{As} 3-\mathrm{O} 11^{\mathrm{iii}}$ & $106.83(13)$ \\
\hline $\mathrm{Na} 1^{\mathrm{i}}-\mathrm{As} 4-\mathrm{Na} 2^{\mathrm{xv}}$ & $145.04(5)$ \\
\hline
\end{tabular}

\begin{tabular}{|c|c|}
\hline $\mathrm{Na} 1^{\mathrm{xi}}-\mathrm{Na} 2-\mathrm{O} 13^{\mathrm{vi}}$ & $77.57(10)$ \\
\hline 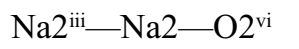 & $132.33(13)$ \\
\hline $\mathrm{Na} 2^{2 \mathrm{ii}}-\mathrm{Na} 2-\mathrm{O} 8^{\mathrm{xi}}$ & $43.30(8)$ \\
\hline $\mathrm{Na} 2^{\mathrm{iii}}-\mathrm{Na} 2-\mathrm{O} 13^{\mathrm{vi}}$ & $130.98(10)$ \\
\hline 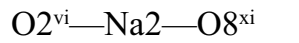 & $145.4(2)$ \\
\hline 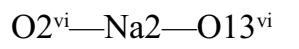 & $81.18(12)$ \\
\hline $\mathrm{O} 8^{\mathrm{xi}}-\mathrm{Na} 2-\mathrm{O} 13^{\mathrm{vi}}$ & $88.13(11)$ \\
\hline As $1-\mathrm{Na} 3-\mathrm{As} 1^{\mathrm{iii}}$ & $115.32(9)$ \\
\hline $\mathrm{As} 1-\mathrm{Na} 3-\mathrm{Na} 1^{\mathrm{xix}}$ & $98.15(10)$ \\
\hline 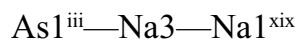 & $98.15(10)$ \\
\hline $\mathrm{Ni1}-\mathrm{O} 1-\mathrm{Ni} 4$ & $125.67(17)$ \\
\hline Ni1-O1-As2 & $123.00(15)$ \\
\hline $\mathrm{Ni4}-\mathrm{O} 1-\mathrm{As} 2$ & $93.49(12)$ \\
\hline $\mathrm{Ni} 2 b^{\mathrm{v}}-\mathrm{O} 2-\mathrm{As} 1$ & $107.3(16)$ \\
\hline $\mathrm{Ni} 2 b^{v}-\mathrm{O} 2-\mathrm{Na} 2^{\text {vii }}$ & $103.8(19)$ \\
\hline $\mathrm{As} 1-\mathrm{O} 2-\mathrm{Na} 2^{\mathrm{vii}}$ & $107.4(2)$ \\
\hline $\mathrm{Ni} 1-\mathrm{O} 3-\mathrm{As} 3$ & $121.8(2)$ \\
\hline $\mathrm{Ni} 3^{\mathrm{vi}}-\mathrm{O} 4-\mathrm{Ni} 4$ & $96.68(11)$ \\
\hline $\mathrm{Ni} 3^{\mathrm{vi}}-\mathrm{O} 4-\mathrm{As} 2^{\mathrm{i}}$ & $124.13(16)$ \\
\hline $\mathrm{Ni} 4-\mathrm{O} 4-\mathrm{As} 2^{\mathrm{i}}$ & $139.19(19)$ \\
\hline $\mathrm{Ni} 3-\mathrm{O} 5-\mathrm{As} 2$ & $129.51(19)$ \\
\hline 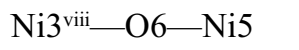 & $104.78(13)$ \\
\hline $\mathrm{Ni}^{\mathrm{viii}}-\mathrm{O} 6-\mathrm{As} 4$ & $121.23(17)$ \\
\hline $\mathrm{Ni5}-\mathrm{O} 6-\mathrm{As} 4$ & $118.91(19)$ \\
\hline As4-O8- $-\mathrm{Na} 1$ & $143.8(2)$ \\
\hline $\mathrm{As} 4-\mathrm{O} 8-\mathrm{Na} 2^{\mathrm{xv}}$ & $105.9(2)$ \\
\hline As4-O8- $-\mathrm{Na} 2^{\mathrm{xvi}}$ & $105.9(2)$ \\
\hline $\mathrm{Na} 1-\mathrm{O} 8-\mathrm{Na} 2^{\mathrm{xv}}$ & $98.65(19)$ \\
\hline $\mathrm{Na} 1-\mathrm{O} 8-\mathrm{Na} 2^{\mathrm{xvi}}$ & $98.65(19)$ \\
\hline $\mathrm{Na} 2^{\mathrm{xv}}-\mathrm{O} 8-\mathrm{Na} 2^{\mathrm{xvi}}$ & $93.40(14)$ \\
\hline $\mathrm{Ni} 3^{\mathrm{ix}}-\mathrm{O} 10-\mathrm{Ni}^{\mathrm{v}}$ & $99.49(11)$ \\
\hline $\mathrm{Ni}^{3{ }^{3 x}}-\mathrm{O} 10-\mathrm{As} 1$ & $132.3(2)$ \\
\hline $\mathrm{Ni}^{\mathrm{v}}-\mathrm{O} 10-\mathrm{As} 1$ & $122.37(14)$ \\
\hline $\mathrm{Ni2} a-\mathrm{O} 11-\mathrm{Ni} 4$ & $121.2(3)$ \\
\hline $\mathrm{Ni2a}-\mathrm{O} 11-\mathrm{As} 3$ & $100.3(3)$ \\
\hline $\mathrm{Ni4}-\mathrm{O} 11-\mathrm{As} 3$ & $128.29(18)$ \\
\hline $\mathrm{Ni4}-\mathrm{O} 12-\mathrm{As} 1$ & $134.0(2)$ \\
\hline Ni5-O13-As2 & $97.57(12)$ \\
\hline $\mathrm{Ni} 5-\mathrm{O} 13-\mathrm{Na} 2^{\mathrm{vii}}$ & $114.05(13)$ \\
\hline As $2-\mathrm{O} 13-\mathrm{Na} 2^{\mathrm{vii}}$ & $142.98(18)$ \\
\hline $\mathrm{Ni}^{\mathrm{xx}}-\mathrm{O} 14-\mathrm{As} 4$ & $133.6(2)$ \\
\hline $\mathrm{Ni} 3-\mathrm{O} 15-\mathrm{Ni} 6^{\mathrm{xxi}}$ & $124.68(17)$ \\
\hline Ni3-O15-As1 & $97.52(13)$ \\
\hline $\mathrm{Ni}^{\mathrm{xxi}}-\mathrm{O} 15-\mathrm{As} 1$ & $120.85(15)$ \\
\hline
\end{tabular}

Symmetry codes: (i) $-x+1, y,-z$; (ii) $-x+1,-y,-z$; (iii) $x,-y, z$; (iv) $-x+1, y,-z+1$; (v) $-x+3 / 2,-y+1 / 2,-z+1$; (vi) $x-1 / 2,-y+1 / 2, z$; (vii) $x+1 / 2,-y+1 / 2, z$; (viii) $-x+3 / 2,-y+1 / 2,-z$; (ix) $-x+2, y,-z+1$; (x) $x-1 / 2, y-1 / 2, z+1$; (xi) $-x+1 / 2, y-1 / 2,-z$; (xii) $x-1, y, z$; (xiii) $-x+1,-y,-z+1$; (xiv) $x-1,-y, z$; (xv) $-x+1 / 2, y+1 / 2,-z$; (xvi) $-x+1 / 2,-y+1 / 2,-z$; (xvii) $x,-y+1, z$; (xviii) $x-1 / 2, y+1 / 2, z$; (xix) $x+1 / 2, y-1 / 2, z$; (xx) $x+1 / 2, y+1 / 2, z-1$; (xxi) $x+1, y, z$. 